\title{
Non-mucinous adenocarcinoma: a unique case with a deceptive presentation
}

\begin{abstract}
Non-mucinous lepidic adenocarcinoma with pneumonic presentation is a rare form of lung cancer. Its ability to mimic infectious and inflammatory diseases in both patient presentation and diagnostic imaging can result in a delayed diagnosis. This case follows the workup of a 60-year-old non-smoker female who immigrated from Ecuador twenty years prior and worked at a shore repair shop for the past two years who presented with worsening shortness of breath and $15 \mathrm{lbs}$ weight loss over a two-month period. A unique aspect of this patient's presentation was that the typical imaging and bronchoscopy findings often seen to distinguish this type of lung adenocarcinoma from other disease processes were absent. No nodular masses were observed on Computed Tomography (CT), and no lesions or airway abnormalities were seen on bronchoscopy. It was not until biopsy and bronchoalveolar lavage (BAL) that the diagnosis of adenocarcinoma was recognized. Our case aims to increase awareness among clinicians of the unpredictable presentation of nonmucinous lepidic adenocarcinoma. Furthermore, we want to highlight the relevance of including adenocarcinoma of the lung in the differential diagnosis even when infectious or occupational etiologies appear more likely.
\end{abstract}

Keywords: oncology, lung cancer, adenocarcinoma, non-mucinous adenocarcinoma, bronchioalveolar carcinoma

\author{
Volume I2 Issue 5 - 202 I
}

\author{
Jason Pannozzo,' Siddharth Vyas,' George \\ W Letterio,' Harold Matos-Casano, ${ }^{2}$ Utpal \\ Bhatt, ${ }^{3}$ Wilhelmina Cruz-Vetrano ${ }^{4}$ \\ 'Medical University of the Americas, Department of Internal \\ Medicine, Wyckoff Heights Medical Center, New York \\ ${ }^{2}$ Department of Internal Medicine, Wyckoff Heights Medical \\ Center, New York \\ ${ }^{3}$ Division of Pulmonary and Critical Care Medicine, Wyckoff \\ Heights Medical Center, New York \\ ${ }^{4}$ Department of Pathology, Wyckoff Heights Medical Center, \\ New York
}

Correspondence: Jason Pannozzo, Medical University of the Americas, Department of Internal Medicine,Wyckoff Heights Medical Center, Brooklyn, New York, Tel (405)819-72I0, Email j.pannozzo@mua.edu

Received: September 15, 2021 | Published: September 28, 2021

\section{Introduction}

Lung cancer is the single largest contributor to cancer-related deaths around the world. ${ }^{1}$ The United States Preventive Services Task Force (USPSTF) recommends annual low dose Computed Tomography (CT) for screening of asymptomatic adults aged 50 to 80 years old with a 20 pack-year smoking history and currently smoke or have quit smoking within the past 15 years. ${ }^{2}$ While cigarettes remain the most significant risk factor for lung cancer, their use in the United States has declined over the last few decades due to public education and tobacco control policies. ${ }^{3}$ In turn, there has been a concomitant shift in the prevalence of different histological subtypes of lung cancer. As the rates of squamous cell and small cell lung cancer decrease, adenocarcinoma has become the predominant histological subtype in the United States. ${ }^{1}$ Considering that patients with lung cancer may present with signs and symptoms that overlap with non-neoplastic respiratory tract illnesses, the diagnosis of cancer in patients may be missed or delayed if more established risk factors are absent from their medical history. ${ }^{4}$ To illustrate this point and increase awareness among clinicians, the authors present a case of lung adenocarcinoma where the diagnosis was recognized only after invasive workup for the evaluation of a presumed inflammatory disease of the lung was pursued.

\section{Case description}

A 60-year-old female presented to our emergency department with a one-month history of worsening shortness of breath, non-productive cough, and $15 \mathrm{lbs}$ weight loss over a period of two months. Past medical history was significant for recurrent episodes of interstitial pneumonia and gastritis, the latter to which she attributed her weight loss. She had been seen in the clinic multiple times over the past six months due to intermittent bouts of cough and dyspnea. Chest X-rays (CXR) on two separate occasions revealed mild bilateral interstitial infiltrates and she was treated empirically for respiratory tract infections. Social history consisted of no tobacco use and employment at a shoe repair shop for the past two years. She immigrated from Ecuador twenty years prior and last visited in 2018. There was no family history of cancer.

On evaluation the patient was afebrile and the physical exam was unremarkable except for a heart rate of $104, \mathrm{SpO}_{2}$ of $94 \%$, and inspiratory crackles on lung auscultation. A Computed Tomography Angiogram (CTA) of the chest revealed no evidence of pulmonary embolism, extensive diffuse airspace opacification bilaterally, worse on the right, and a loculated left pleural effusion. Laboratory studies including complete blood count, metabolic panel, brain natriuretic peptide, troponins, thyroid stimulating hormone, and blood cultures were unremarkable. Arterial blood gas on room air showed $\mathrm{pH}$ 7.44, $\mathrm{PaO}_{2} 65 \mathrm{mmHg}, \mathrm{PaCO}_{2} 39 \mathrm{mmHg}$, and $\mathrm{HCO}_{3} 26.5 \mathrm{mEq} / \mathrm{L}$. The patient was placed on supplemental oxygen and treated empirically with vancomycin, ceftriaxone, and doxycycline. Autoimmune and interstitial lung diseases were also considered in the differential.

Image-guided thoracentesis yielded 20cc of exudative fluid (LDH: 125; protein: 3.90 ). Cytology and microbiology studies of the fluid were negative. QuantiFERON was positive and three consecutive Acid-Fast stains and cultures were negative consistent with latent tuberculosis. Additional tests included influenza, HIV, urine legionella and pneumococcal antigen tests, ANA, P-ANCA, and C-ANCA all of which were negative. In view of these findings fiberoptic bronchoscopy was performed. The airway was clear with minimal secretions and no erythema or endobronchial lesions were noted. Bronchoalveolar lavage (BAL) and transbronchial biopsy of the right superior and inferior lobes revealed the diagnosis. Cytology of BAL fluid was positive for malignant cells showing clusters of atypical glandular epithelial cells consistent with adenocarcinoma. Results were confirmed by the biopsy with the final diagnosis being nonmucinous adenocarcinoma with lepidic growth and micropapillary features. Antibiotics were discontinued upon confirmation of the 
diagnosis and subsequent imaging helped to conclude that she had probable stage IV disease. Molecular analysis showed no evidence of mutations in EGFR or ALK/ROS1 translocations. The patient was instructed to follow up treatment at a tertiary care hospital and was discharged with codeine syrup for cough and home oxygen due to persistent hypoxemia decreasing to $87 \%$ on exertion. At outpatient follow-up the patient declined any further intervention and stated that she was moving back home to Ecuador.

\section{Discussion}

The World Health Organization (WHO) in 2015 published the World Health Organization Classification of Tumors of the Lung, Pleura, Thymus and Heart in response to the expansion of medical knowledge regarding these types of neoplasia. In particular, the histological subtyping of adenocarcinomas of the lung led to the additional sub-classification of these tumors as lepidic, papillary, acinar, micropapillary and solid. The lepidic pattern of adenocarcinoma, a noninvasive growth pattern, was formerly called bronchioalveolar carcinoma (BAC). ${ }^{5}$ Lepidic growth refers to the spread of the tumor cells along the pre-existing structures of the alveoli. Sclerotic thickening of the alveolar septa may also be observed. ${ }^{6}$

Clinical features of patients with lepidic pattern adenocarcinoma may present with a wide array of symptoms. Weight loss and weakness have been observed in approximately $34.0 \%$ of patients, while coughing and dyspnea range from $45-54.7 \%$ and $37-45 \%$, respectively. ${ }^{7}$ In addition, chest pain and hemoptysis occurred in upwards of $27.0 \%$ of patients diagnosed with lepidic adenocarcinoma. ${ }^{7}$ On imaging, chest x-ray will often reveal a coin-like lesion in the lung fields. ${ }^{4}$ Further evaluation through CT scan will show the presence of ground glass lesions and multiple sub-solid nodules. It is through these findings that the International Association for the Study of Lung Cancer (IASLC) established the physical and clinical criteria of lepidic adenocarcinoma. ${ }^{8}$

Adenocarcinoma of the lung can also present with infiltrates on imaging that resembles an infectious or inflammatory process. This uncommon presentation, known as pneumonic adenocarcinoma, has been noted to occur in only approximately $8.9 \%$ of all adenocarcinomas of the lungs. ${ }^{9}$ With adenocarcinoma composing $38.5 \%$ of lung cancers, pneumonic type of adenocarcinoma may be present in less than $3.43 \%$ of all lung cancers. ${ }^{10}$ This unique presentation can be further differentiated as mucinous or non-mucinous on histological evaluation. Non-mucinous has been observed in $42 \%$ of pneumonic adenocarcinomas, while mucinous is found in $45 \%$ with the remaining presentations displaying a mixed histological finding. ${ }^{11}$

Therefore, the patient's diagnosis of lepidic non-mucinous adenocarcinoma with a pneumonic presentation is in itself an unusual finding, comprising approximately $1.5 \%$ of all lung cancer cases. More importantly, however, this case emphasizes the need to include primary lung adenocarcinomas, specifically the pneumonic type, as a differential diagnosis when clinical findings are suggestive of an infectious or inflammatory disease of the lungs.

Non-mucinous adenocarcinoma with lepidic histological findings and a pneumonic presentation is an uncommon finding with limited patient literature. In addition, this patient's uncharacteristic non-mucinous adenocarcinoma, is capable of displaying clinical features similar to that of infectious or inflammatory diseases. ${ }^{11}$ These deceptive clinical and radiological findings make early recognition and a timely diagnosis of pneumonic type non-mucinous adenocarcinoma challenging. Notable features of this case begin with the initial presentation of the patient with a persistent cough followed by dyspnea, and atypical CT findings. The CT scan consisted of extensive diffuse airspace opacification within the upper and lower lobes bilaterally, left lower lobe consolidation and a loculated left pleural effusion. In accordance with recent studies comparing pneumonic type adenocarcinoma to infectious pneumonia, peripheral consolidation with surrounding nodules favored the diagnosis of pneumonic-type adenocarcinoma. ${ }^{11}$ The lack of any nodular masses in the patient's imaging is a deceptive finding, and resembles an infectious pneumonia. In addition, another distinctive aspect of the patient's presentation was the rapid extension of the cancer to involve both lung fields in three months. This rate of progression is inconsistent with the typical presentation of lepidic adenocarcinoma. Tumors that display lepidic growth with minimal or mild thickening of the alveolar septa, as observed in this case, have an extremely favorable prognosis with a 5-year survival rate of $95 \% .{ }^{12}$ The aggressive involvement of both lung fields by this patient's cancer suggests that further investigation is required into the growth rate and progression of pneumonic type adenocarcinomas. There is also an etiologic relationship between lung cancer and scarring. ${ }^{13}$ This raises the question if occupational exposure to hazardous chemicals at the shoe repair shop or her latent tuberculosis could have been contributing factors to the origin or progression of her cancer.

Bronchoscopy also initially yielded some unexpected results. Pneumonic infections produce an inflammatory response with physiological characteristics of erythema upon bronchoscopic evaluation. ${ }^{14}$

Direct visualization of the patient's lungs, however, did not display these expected features. Instead, no endobronchial lesions or airway abnormalities were seen with only minimal secretions noted. Additionally, when comparing characteristic bronchoscopy findings of adenocarcinoma to this patient's presentation several features were absent. These included an endobronchial mass, mucosal infiltration, carina widening, and mucosal hyperemia. ${ }^{15}$ Adenocarcinoma are more commonly found in distal parts of the bronchus not directly visible through bronchoscopy. Although up to $40 \%$ of non-visible lung tumors are adenocarcinomas, signs of mass effect such as external compression and luminal narrowing may still be evident. ${ }^{15}$ These were not observed during the patient's bronchoscopy. A definitive diagnosis of non-mucinous adenocarcinoma was only recognized after biopsy of peripheral lobe segments with the aid of fluoroscopy. Cytologic evaluation of the BAL confirmed the diagnosis by demonstrating clusters of atypical glandular epithelial cells. This reinforces the importance of flexible bronchoscopy in differentiating a pneumonic infection from non-mucinous adenocarcinoma with pneumonic presentation and the need for biopsy and BAL in the diagnosis of lung cancer.

\section{Conclusion}

Non-mucinous lepidic adenocarcinoma with pneumonic presentation is an uncommon form of lung cancer. Due to its uncanny ability to mimic both infectious and inflammatory diseases in patient presentation and on diagnostic imaging can result in a prolonged period of time to establish a diagnosis. This atypical presentation includes a progressively worsening cough with diffuse bilateral infiltrates on CXR and CT imaging that is refractory to antimicrobial therapy. This case was further complicated by the fact that no nodular masses were seen on CT, which often aids to differentiate this cancer from an infectious process. On bronchoscopy no lesions or airway abnormalities were observed and it was not until biopsy and BAL that the diagnosis of adenocarcinoma was recognized. This emphasizes the necessity for clinicians to include adenocarcinoma of the lung in the 
differential diagnosis even when infectious or occupational etiologies appear more likely. This is especially true when radiographic imaging is suggestive of an inflammatory disease but the lungs are clear on direct visualization through bronchoscopy. Ultimately, this will result in an earlier diagnosis of cancer and a better prognosis for the patient.

\section{Acknowledgments}

None.

\section{Conflicts of interest}

Authors declare that there is no conflict of interest.

\section{References}

1. Barta JA, Powell CA, Wisnivesky JP. Global epidemiology of lung cancer. Annals of Global Health. 2019;85(1):8.

2. Krist AH, Davidson KW, Mangione CM, et al. Screening for lung cancer: US preventive services task force recommendation statement. JAMA. 2021;325(10):962-970.

3. de Groot PM, Wu CC, Carter BW, et al. The epidemiology of lung cancer. Translational Lung Cancer Research. 2018;7(3):220-223.

4. Morikawa S, Okamura T, Yamaguchi T, et al. Clinical features of primary lung cancer presenting as pulmonary consolidation mimicking pneumonia. Fujita Medical Journal. 2016;2(1):17-21.

5. Kadota K, Villena-Vargas J, Yoshizawa A, et al. Prognostic significance of adenocarcinoma in situ, minimally invasive adenocarcinoma, and nonmucinous lepidic predominant invasive adenocarcinoma of the lung in patients with stage I disease. The American Journal of Surgical Pathology. 2014;38(4):448-460.

6. Zheng M. Classification and pathology of lung cancer. Surgical Oncology Clinics. 2016;25(3):447-468.
7. Jiménez-Zarazúa O, Vélez-Ramírez LN, Padilla-López JC, et al. Invasive Pulmonary Adenocarcinoma with Lepidic Growth Pattern in a Pregnant Patient. Case Reports in Oncology. 2018;11(3):822-834.

8. Detterbeck FC, Marom EM, Arenberg DA, et al. The IASLC lung cancer staging project: background data and proposals for the application of TNM staging rules to lung cancer presenting as multiple nodules with ground glass or lepidic features or a pneumonic type of involvement in the forthcoming eighth edition of the TNM classification. Journal of Thoracic Oncology. 2016;11(5):666-680.

9. Becnel D, Biswas A, Sriram PS. Pneumonic Adenocarcinoma: Presentation and Outcomes. Thoracic Oncology Clinical Outcomes. American Thoracic Society; 2017. A4591-A4591 p.

10. Cruz CS, Tanoue LT, Matthay RA. Lung cancer: epidemiology, etiology, and prevention. Clinics in Chest Medicine. 2011;32(4):605-644.

11. Daoud A, Laktineh A, El Zein S, et al. Unusual presentation of primary lung adenocarcinoma mimicking pneumonia: Case report and literature review. Respiratory Medicine Case Reports. 2019;28:100881.

12. Noguchi M. Stepwise progression of pulmonary adenocarcinomaclinical and molecular implications. Cancer and Metastasis Reviews. 2010;29(1):15-21.

13. Ahmed F, Yasin H, Mohamed HE. A case of lung adenocarcinoma originating from an old posttraumatic scar in a young patient. Case Reports in Oncological Medicine. 2019;2019:8395389.

14. Soyer T. The role bronchoscopy in the diagnosis of airway disease in children. Journal of Thoracic Disease. 2016;8(11):3420-3426.

15. Rabahi MF, Ferreira AA, Reciputti BP, et al. Fiberoptic bronchoscopy findings in patients diagnosed with lung cancer. Journal Brasileiro de Pneumologia. 2012;38(4):445-451. 